\title{
Collisions of two breathers at the surface of deep water
}

\author{
A. I. Dyachenko ${ }^{1,3}$, D. I. Kachulin ${ }^{1}$, and V. E. Zakharov ${ }^{1,3,2,4}$ \\ ${ }^{1}$ Novosibirsk State University, Pirogova 2, Novosibirsk-90, 630090, Russia \\ ${ }^{2}$ Department of Mathematics, University of Arizona, Tucson, AZ, 857201, USA \\ ${ }^{3}$ Landau Institute for Theoretical Physics, 1-a Semenova pr., Chernogolovka, Moscow Region, 142432, Russia \\ ${ }^{4}$ Physical Institute of RAS, Leninskiy prospekt, 53, Moscow, 119991, Russia \\ Correspondence to: A. I. Dyachenko (alexd@itp.ac.ru)
}

Received: 3 June 2013 - Published in Nat. Hazards Earth Syst. Sci. Discuss.: 28 June 2013

Revised: 5 November 2013 - Accepted: 7 November 2013 - Published: 9 December 2013

\begin{abstract}
We present results of numerical experiments on long-term evolution and collisions of breathers (which correspond to envelope solitons in the NLSE approximation) at the surface of deep ideal fluid. The collisions happen to be nonelastic. In the numerical experiment it can be observed only after many acts of interactions. This supports the hypothesis of "deep water nonintegrability". The experiments were performed in the framework of the new and refined version of the Zakharov equation free of nonessential terms in the quartic Hamiltonian. Simplification is possible due to exact cancellation of nonelastic four-wave interaction.
\end{abstract}

\section{Introduction}

Theory of weakly nonlinear waves on shallow water is a nursery for several completely integrable models. Among them are the famous KdV and KP equations (Gardner et al., 1967; Kadomtsev and Petviashvili, 1973; Zakharov and Shabat, 1979), the Boussinesq equation (Zakharov, 1974), and the Kaup system (Kaup, 1975). Detailed study of these integrable systems has not only theoretical, but also practical importance. Recently A. Osborne showed (Osborne, 2010) that representation of solutions of KP equations in the form of Jacobi theta functions is a very efficient and economical way of analyzing experimental data for long waves in coastal areas.

Now the fundamental question appears - what can be done in the case of deep fluid? So far only one integrable model on deep water is known. It is the focusing nonlinear Schrödinger equation describing weakly nonlinear quasimonochromatic wave trains (Zakharov, 1968; Zakharov and Shabat, 1972). Exact solutions of this equation can also be given by theta functions (Belokolos et al., 1994). They are actively used now for determination of freak wave statistics (Osborne, 2010). However, the NLSE has a limited area of application and can hardly be applicable to many experimental situations.

Hopes that the exact Euler equation for potential flow on deep water with free surface in the presence of gravity is integrable appeared in 1994 when two of us (Dyachenko and Zakharov, 1994) established that the coefficient of a scattering matrix connecting asymptitics at $t \rightarrow \pm \infty$ states of wave field, corresponding to inelastic four-wave processes and governed by resonant conditions

$k+k_{1}=k_{2}+k_{3} \quad \omega_{k}+\omega_{k_{1}}=\omega_{k_{2}}+\omega_{k_{3}}$,

where

$\omega_{k}=\sqrt{g|k|}$

in 1-D geometry is identically equal to zero.

However, this cancellation is just a weak necessary condition for integrability and is far from being sufficient. For integrability in "strong sense" we need cancellation in all orders of perturbation theory (see Zakharov and Schulman, 1991). However, in Dyachenko et al. (1995) it was shown that not all members of a five-wave scattering matrix are zero, thus we can only hope for integrability in some "weak sense". We will not discuss this subject having a "strong mathematical flavor" here.

Meanwhile, efficient methods for numerical simulations of the exact Euler equation were developed during the last decade; massive numerical experiments were also performed. Again, some of them can be considered as a certain indication of integrability. 
In the framework of NLSE approximation there is an exact solution - envelope soliton. Do such solutions exist in the exact Euler equation? If the system is nonintegrable, the soliton exists only during a finite time; then it must lose its energy due to radiation in a backward direction (Zakharov and Kuznetsov, 1998). In the nonintegrable MMT model this backward radiation is a very strong effect leading to the formation of an "abnormal" weak turbulent spectrum (Rumpf et al., 2009). However, in our experiments on propagation of steep envelope solitons in the frame of the Euler equation, we did not trace the slightest backward radiation (Dyachenko and Zakharov, 2008). The soliton persistently existed during thousands of their periods.

In this article we present new numerical results shedding some light on the integrability of the deep-water hydrodynamics. We study collisions of breathers (solitons) in the framework of a newly derived approximate equation applicable for small-amplitude waves with any spectral band width. Actually, this is what is called the "Zakharov equation" (see Zakharov, 1968), improved by the implementation of additional canonical transformation to the Poincare normal form. This transformation is possible only due to the still mysterious fact of four-wave interaction cancellation.

The new equation (described in detail in Dyachenko and Zakharov, 2011, 2012) is very convenient for numerical simulations. It has a nice solitonic solution that so far cannot be found analytically, but can be easily obtained numerically. Existence of solitonic solutions and their elastic collisions are indications of integrability. However, just indications are not enough. In this paper we study the collision of such solitons and show that this collision is nonelastic. One can however only see it after multiple collisions. We can interpret this fact as a numerical proof of nonintegrability, at least for this "refined Zakharov equation"1.

\section{Compact equation}

A one-dimensional potential flow of an ideal incompressible fluid with a free surface in a gravity field fluid is described by the following set of equations:

$$
\begin{aligned}
\phi_{x x}+\phi_{z z} & =0 \quad\left(\phi_{z} \rightarrow 0, z \rightarrow-\infty\right), \\
\eta_{t}+\eta_{x} \phi_{x} & =\left.\phi_{z}\right|_{z=\eta} \\
\phi_{t}+\frac{1}{2}\left(\phi_{x}^{2}+\phi_{z}^{2}\right)+g \eta & =\left.0\right|_{z=\eta} ;
\end{aligned}
$$

here $\eta(x, t)$ is the shape of a surface, $\phi(x, z, t)$ is a potential function of the flow and $g$ is gravitational acceleration. As was shown in Zakharov (1968), the variables $\eta(x, t)$ and $\psi(x, t)=\left.\phi(x, z, t)\right|_{z=\eta}$ are canonically conjugated, and

\footnotetext{
${ }^{1}$ Some of the numerical results were put in Dyachenko et al. (2012)
}

satisfy the equations

$$
\frac{\partial \psi}{\partial t}=-\frac{\delta H}{\delta \eta} \quad \frac{\partial \eta}{\partial t}=\frac{\delta H}{\delta \psi} .
$$

Here the Hamiltonian can be written as an infinite series (see Zakharov, 1968):

$$
\begin{aligned}
H & =\frac{1}{2} \int g \eta^{2}+\psi \hat{k} \psi \mathrm{d} x-\frac{1}{2} \int\left\{(\hat{k} \psi)^{2}-\left(\psi_{x}\right)^{2}\right\} \eta \mathrm{d} x+ \\
& +\frac{1}{2} \int\left\{\psi_{x x} \eta^{2} \hat{k} \psi+\psi \hat{k}(\eta \hat{k}(\eta \hat{k} \psi))\right\} \mathrm{d} x+\ldots
\end{aligned}
$$

In this article we consider Hamiltonians up to the fourth order. In the articles (Dyachenko and Zakharov, 2011, 2012) we applied canonical transformation to the Hamiltonian variables $\psi$ and $\eta$ to introduce the normal canonical variable $b(x, t)$. This transformation explicitly exploits the vanishing of four-wave interaction and possibility to consider surface waves moving in the same direction. Briefly, this transformation consists of two steps. First, we introduce normal complex variables $a_{k}(t)$ as follows:

$\eta_{k}=\sqrt{\frac{\omega_{k}}{2 g}}\left(a_{k}+a_{-k}^{*}\right) \quad \psi_{k}=-i \sqrt{\frac{g}{2 \omega_{k}}}\left(a_{k}-a_{-k}^{*}\right)$.

Then one applies transformation from variables $a_{k}$ to $b_{k}$ to exclude nonresonant cubic terms along with nonresonant fourth-order terms. This transformation up to accuracy $O\left(b^{5}\right)$ has the form (Zakharov, 1968; Zakharov et al., 1992):

$$
\begin{aligned}
a_{k} & =b_{k}+\int \Gamma_{k_{1} k_{2}}^{k} b_{k_{1}} b_{k_{2}} \delta_{k-k_{1}-k_{2}} \mathrm{~d} k_{1} \mathrm{~d} k_{2}- \\
& -2 \int \Gamma_{k k_{1}}^{k_{2}} b_{k_{1}}^{*} b_{k_{2}} \delta_{k+k_{1}-k_{2}} \mathrm{~d} k_{1} \mathrm{~d} k_{2}+ \\
& +\int \Gamma_{k k_{1} k_{2}} b_{k_{1}}^{*} b_{k_{2}}^{*} \delta_{k+k_{1}+k_{2}} \mathrm{~d} k_{1} \mathrm{~d} k_{2} \\
& +\int B_{k k_{1}}^{k_{2} k_{3}} b_{k_{1}}^{*} b_{k_{2}} b_{k_{3}} \delta_{k+k_{1}-k_{2}-k_{3}} \mathrm{~d} k_{1} \mathrm{~d} k_{2} \mathrm{~d} k_{3}+ \\
& +\int C_{k k_{1} k_{2}}^{k_{3}} b_{k_{1}}^{*} b_{k_{2}}^{*} b_{k_{3}} \delta_{k+k_{1}+k_{2}-k_{3}} \mathrm{~d} k_{1} \mathrm{~d} k_{2} \mathrm{~d} k_{3} \\
& +\int S_{k k_{1} k_{2} k_{3}} b_{k_{1}}^{*} b_{k_{2}}^{*} b_{k_{3}}^{*} \delta_{k+k_{1}+k_{2}+k_{3}} \mathrm{~d} k_{1} \mathrm{~d} k_{2} \mathrm{~d} k_{3} .
\end{aligned}
$$

The particular choice of coefficients in Eq. (2) is described in Dyachenko and Zakharov $(2011,2012)$. The choice of $\Gamma_{k_{1} k_{2}}^{k}$ and $\Gamma_{k k_{1} k_{2}}$ provides cancellation of cubic terms, while the choice of $C_{k k_{1} k_{2}}^{k_{3}}$ and $S_{k k_{1} k_{2} k_{3}}$ provides cancellation of the nonresonant fourth-order term. The particular choice of $B_{k k_{1}}^{k_{2} k_{3}}$ allows selfconsistent consideration of waves moving in the same direction only, making the Hamiltonian very simple at the same time. For this variable $b(x, t)$, Hamiltonian (1) acquires the nice and elegant form ${ }^{2}$ :

$\mathcal{H}=\int b^{*} \hat{\omega}_{k} b \mathrm{~d} x+\frac{1}{2} \int\left|\frac{\partial b}{\partial x}\right|^{2}\left[\frac{i}{2}\left(b \frac{\partial b^{*}}{\partial x}-b^{*} \frac{\partial b}{\partial x}\right)-\hat{K}|b|^{2}\right] \mathrm{d} x$.

\footnotetext{
${ }^{2}$ There was a misprint in the articles (Dyachenko and Zakharov, 2011, 2012): the coefficient for the quartic term in the Hamiltonian must be $\frac{1}{2}$ instead of $\frac{1}{4}$
} 


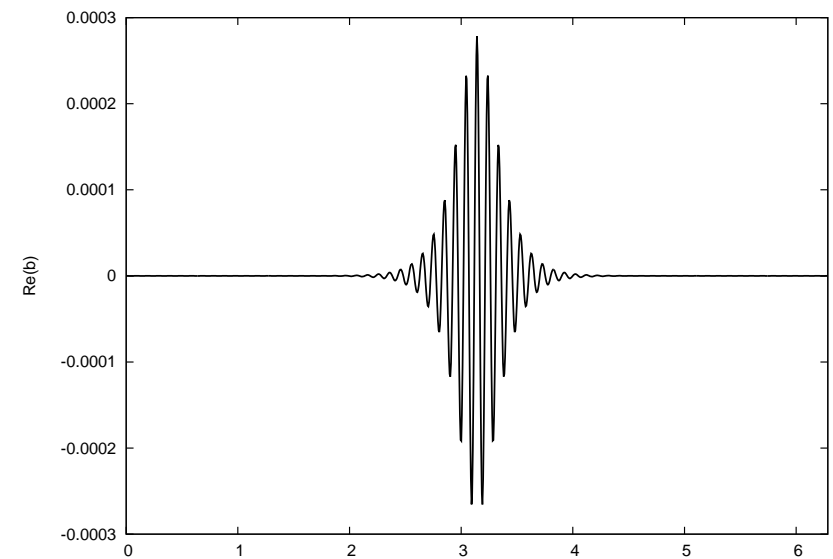

Fig. 1. Real part of $b(x)$ with $V=1 / 16$ and $\Omega=4.01$.

In $K$-space the Hamiltonian has the form:

$$
\begin{aligned}
\mathcal{H} & =\int \omega_{k}\left|b_{k}\right|^{2} \mathrm{~d} k+ \\
& +\frac{1}{2} \int \tilde{T}_{k_{1} k_{2}}^{k_{3} k_{4}} b_{k_{1}}^{*} b_{k_{2}}^{*} b_{k_{3}} b_{k_{4}} \delta_{k_{1}+k_{2}-k_{3}-k_{4}} \mathrm{~d} k_{1} \mathrm{~d} k_{2} \mathrm{~d} k_{3} \mathrm{~d} k_{4}
\end{aligned}
$$

Here

$$
\begin{aligned}
\tilde{T}_{k_{2} k_{3}}^{k k_{1}} & = \\
& \frac{\theta(k) \theta\left(k_{1}\right) \theta\left(k_{2}\right) \theta\left(k_{3}\right)}{8 \pi}\left[\left(k k_{1}\left(k+k_{1}\right)+k_{2} k_{3}\left(k_{2}+k_{3}\right)\right)-\right. \\
& -\left(k k_{2}\left|k-k_{2}\right|+k k_{3}\left|k-k_{3}\right|+k_{1} k_{2}\left|k_{1}-k_{2}\right|+\right. \\
& \left.\left.+k_{1} k_{3} \mid k_{1}-k_{3}\right)\right],
\end{aligned}
$$

$\theta(k)= \begin{cases}0, & \text { if } k \leq 0 \\ 1, & \text { if } k>0\end{cases}$

The Fourier transform is defined as follows:

$b(x)=\frac{1}{\sqrt{2 \pi}} \int_{-\infty}^{\infty} b_{k} e^{i k x} \mathrm{~d} x$,

where $b(x)$ can be analytically continued to $x+i y, y>0$. The motion equation for $b_{k}$ should be understood as follows:

$i \frac{\partial b}{\partial t}=\hat{P}^{+} \frac{\delta \mathcal{H}}{\delta k_{k}^{*}}$,

here $\hat{P}^{+}$- projection operator to the upper half-plane.

$\hat{P^{+2}}=\hat{P}^{+}=\frac{1}{2}(1-i \hat{H})$.

This operator is the consequence of $\theta$ functions in Eq. (4). It keeps only positive $k$ in the system of waves. (So, we

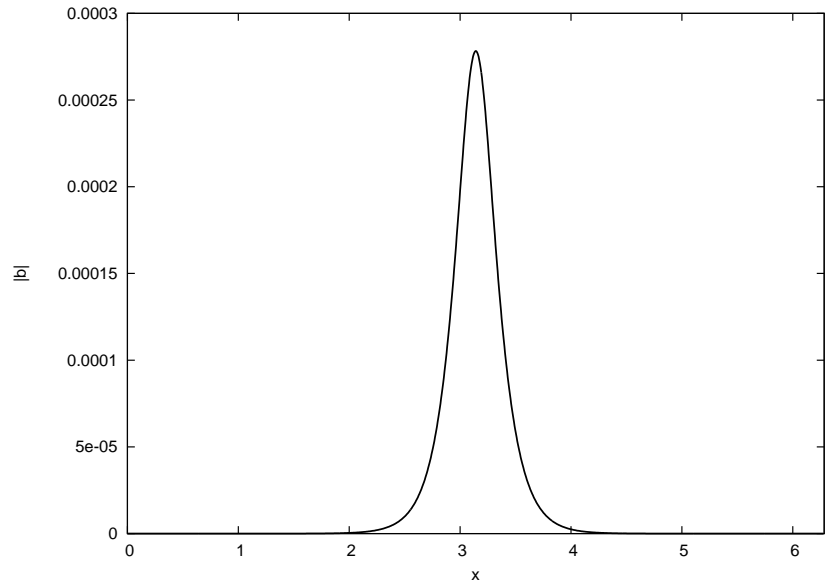

Fig. 2. Modulus of $b(x)$ with $V=1 / 16$ and $\Omega=4.01$.

consider self-consistent systems of waves propagating in the same direction.)

The corresponding equation of motion is the following:

$$
\begin{aligned}
i \frac{\partial b}{\partial t}=\hat{\omega}_{k} b+ & \frac{i}{4} \hat{P}^{+}\left[b^{*} \frac{\partial}{\partial x}\left(b^{\prime 2}\right)-\frac{\partial}{\partial x}\left(b^{* \prime} \frac{\partial}{\partial x} b^{2}\right)\right]- \\
& -\frac{1}{2} \hat{P}^{+}\left[b \cdot \hat{K}\left(\left|b^{\prime}\right|^{2}\right)-\frac{\partial}{\partial x}\left(b^{\prime} \hat{K}\left(|b|^{2}\right)\right)\right],
\end{aligned}
$$

or in $K$-space

$i \frac{\partial b_{k}}{\partial t}=\omega_{k} b_{k}+\int \tilde{T}_{k k_{1}}^{k_{2} k_{3}} b_{k_{1}}^{*} b_{k_{2}} b_{k_{3}} \delta_{k+k_{1}-k_{2}-k_{3}} \mathrm{~d} k_{1} \mathrm{~d} k_{2} \mathrm{~d} k_{3}$.

\section{Breathers and numerical simulation of its collisions}

A breather is the localized solution of Eq. (6) of the following type:

$b(x, t)=B(x-V t) e^{i\left(k_{0} x-\omega_{0} t\right)}$,

where $k_{0}$ is the wavenumber of the carrier wave, $V$ is the group velocity and $\omega_{0}$ is the frequency close to $\omega_{k_{0}}$. In the Fourier space a breather can be written as follows:

$b_{k}(t)=e^{-i(\Omega+V k) t} \phi_{k}$,

where $\Omega$ is close to $\frac{\omega_{k_{0}}}{2}$.

For $\phi_{k}$ the following equation is valid:

$\left(\Omega+V k-\omega_{k}\right) \phi_{k}=\int \tilde{T}_{k k_{1}}^{k_{2} k_{3}} \phi_{k_{1}}^{*} \phi_{k_{2}} \phi_{k_{3}} \delta_{k+k_{1}-k_{2}-k_{3}} \mathrm{~d} k_{1} \mathrm{~d} k_{2} \mathrm{~d} k_{3}$.

One can treat $\phi_{k}$ as a pure real function of $k$.

To solve Eq. (10), one can use the Petviashvili iteration method (Petviashvili, 1976; Lakoba and Yang, 2007) ( $n$ is the number of iterations):

$$
\begin{aligned}
& \left(\Omega+V k-\omega_{k}\right) \phi_{k}^{n+1}= \\
& \quad M^{n} \int \tilde{T}_{k k_{1}}^{k_{2} k_{3}} \phi_{k_{1}}^{* n} \phi_{k_{2}}^{n} \phi_{k_{3}}^{n} \delta_{k+k_{1}-k_{2}-k_{3}} \mathrm{~d} k_{1} \mathrm{~d} k_{2} \mathrm{~d} k_{3} .
\end{aligned}
$$




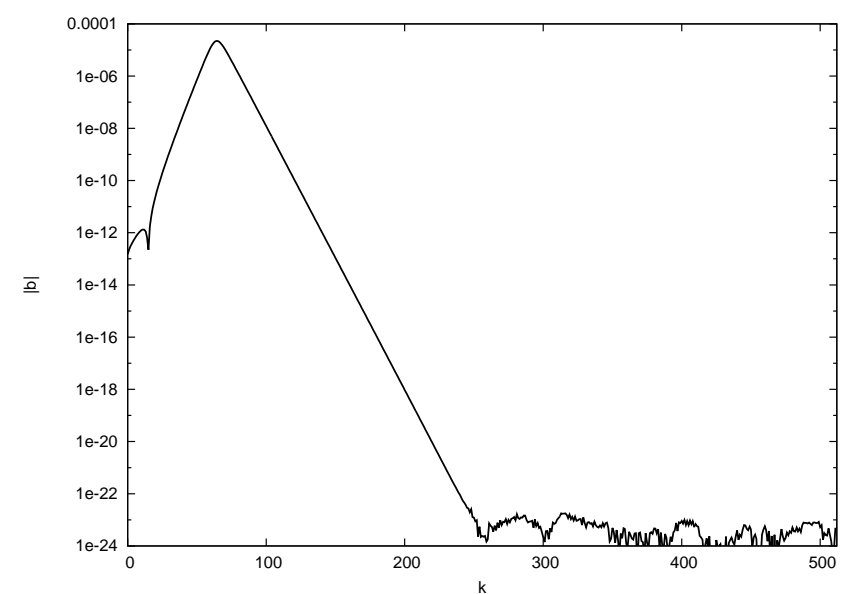

Fig. 3. Spectrum of $b(x)$ with $V=1 / 16$ and $\Omega=4.01$.

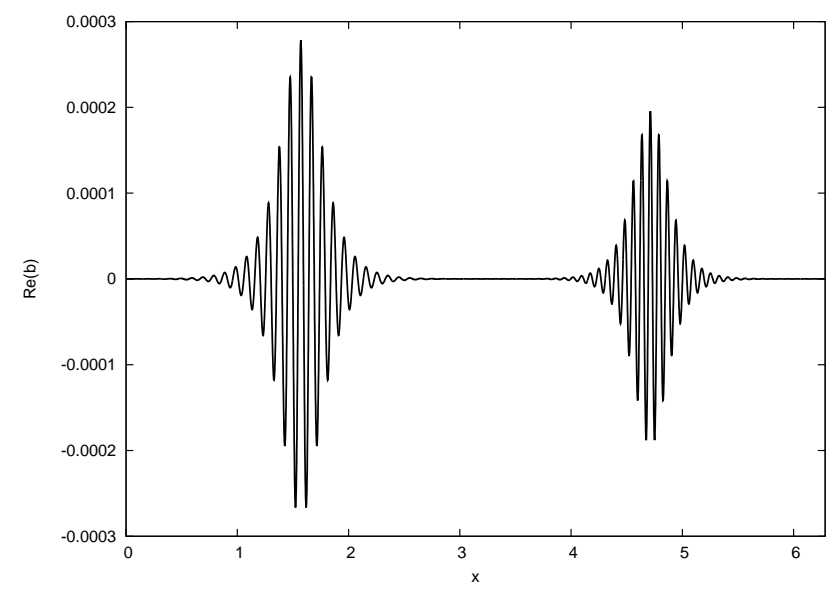

Fig. 4. Initial condition with two breathers.

The Petviashvili coefficient $M^{n}$ is the following:

$M^{n}=\left[\frac{<\phi_{k}^{n}\left(\Omega+V k-\omega_{k}\right) \phi_{k}^{n}>}{<\phi_{k}^{n} \int \tilde{T}_{k k_{1}}^{k_{2} k_{3}} \phi_{k_{1}}^{* n} \phi_{k_{2}}^{n} \phi_{k_{3}}^{n} \delta_{k+k_{1}-k_{2}-k_{3}} \mathrm{~d} k_{1} \mathrm{~d} k_{2} \mathrm{~d} k_{3}>}\right]^{\frac{3}{2}}$.

The angular brackets mean integration over $k$.

Below we present a typical numerical solution of Eq. (10). Calculations were made in the periodic domain $2 \pi$ with carrier wavenumber $k_{0} \sim 64, V=1 / 16$ and $\Omega=4.01$. In Figs. 1, 2, 3 one can see the real part of $b(x)$, the modulus of $b(x)$ and the Fourier spectrum of $b(x)$. The modulus of $b(x)$ coincides with the modulus of $B(x-V t)$ in Eq. (8) and is similar to the wave envelope if we derive the nonlinear Schrödinger equation from Eq. (6).

To analyze the question about the integrability of Eq. (6), one can consider collision of breathers. It might be elastic or nonelastic. In the papers (Dyachenko et al., 2012; Fedele and Dutykh, 2012) one collision of two breathers was considered.

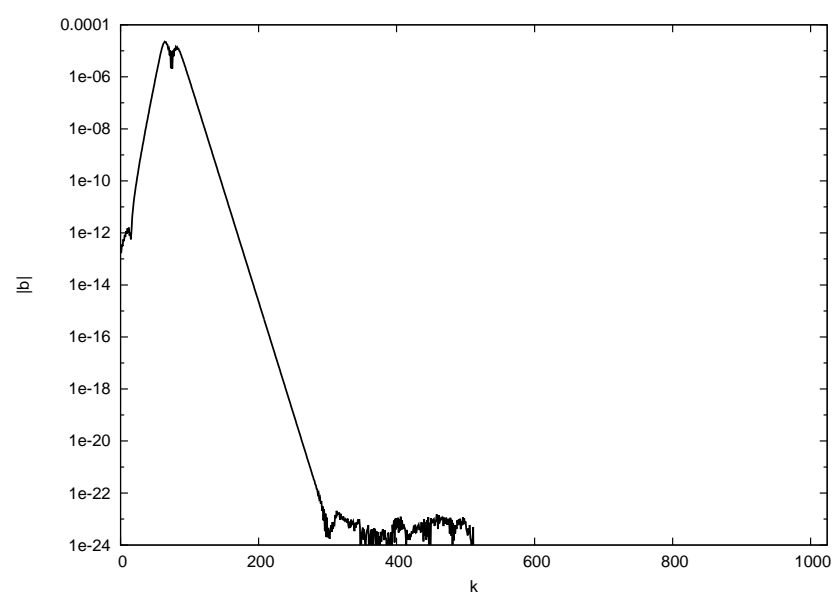

Fig. 5. Initial Fourier spectrum $\left|b_{k}\right|$ of two breathers.

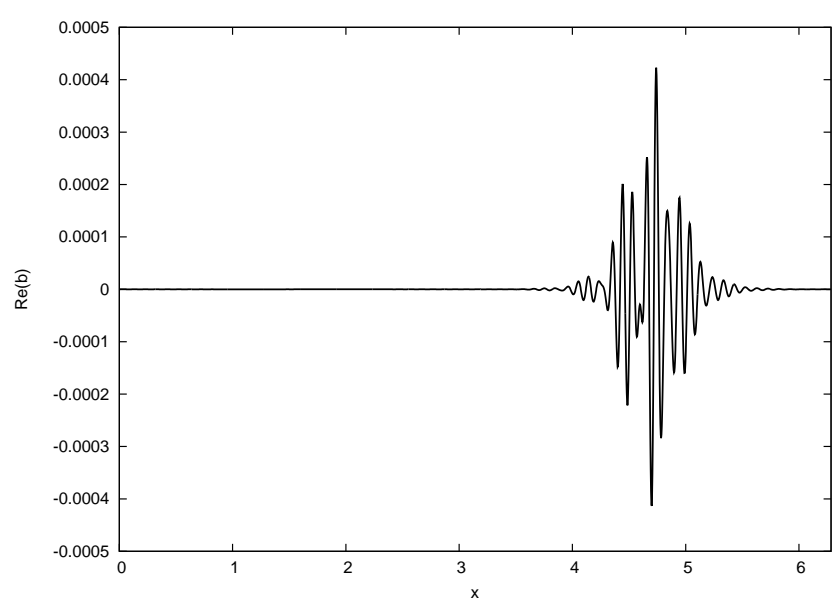

Fig. 6. Two breathers collide.

This collision seemed to be elastic. Here, in this paper, we consider multiple collisions to check integrability numerically. For time-integration schemes, the 4-th order RungeKutta method was used. The scheme is very robust and allows long-term simulation.

To study breather collisions, we performed the following numerical simulation:

- As the initial condition we have used two breathers separated in space (distance was equal to $\pi$.)

- The first breather has the following parameters: $\Omega_{1}=$ $4.01, V_{1}=1 / 16$. The carrier wave number appears to be $\sim 64$.

- For the second breather, $\Omega_{2}=4.51, V_{2}=1 / 18$. The carrier wave number appears to be $\sim 81$.

This initial condition is shown in Fig. 4. Its Fourier spectrum is shown in Fig. 5. 


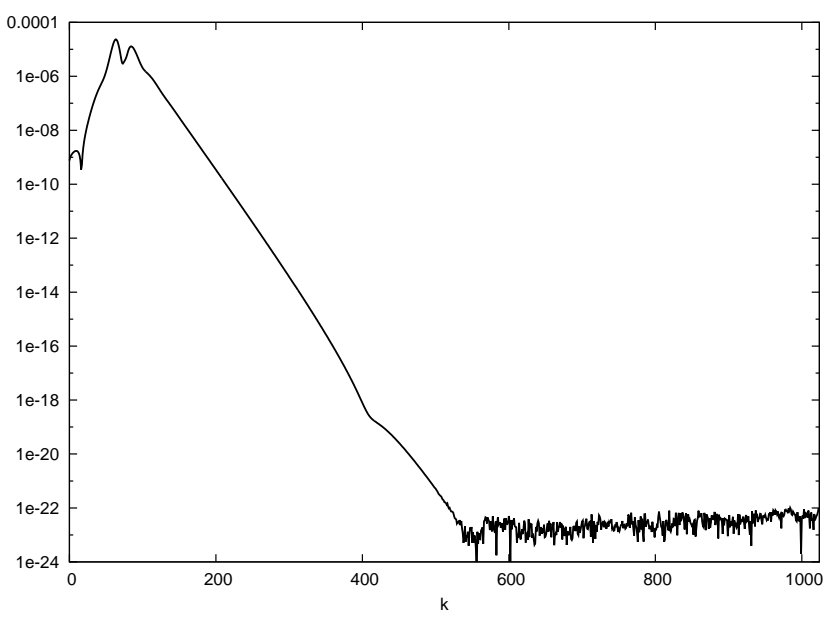

Fig. 7. Fourier spectrum $\left|b_{k}\right|$ at the moment of first collision.

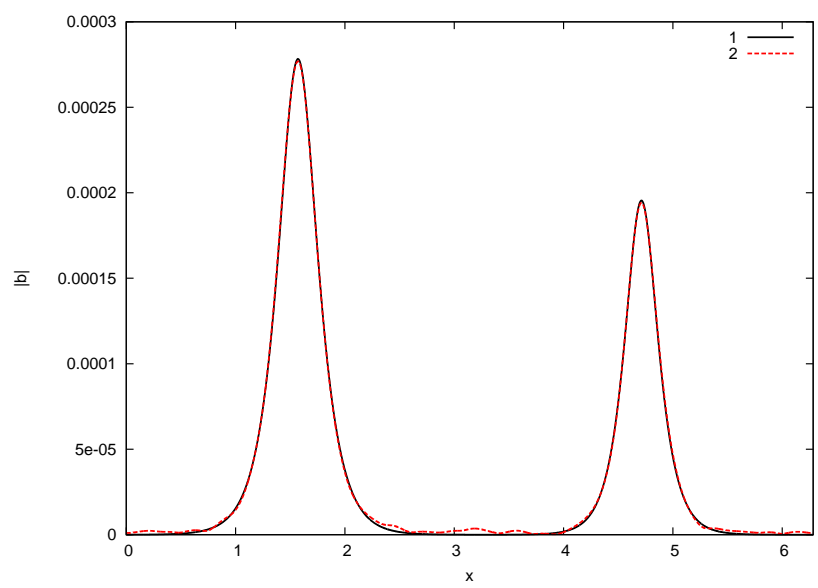

Fig. 8. Modulus of $b(x)$ for two points in time. The solid line corresponds to the initial statement $(t=0)$, the dashed line to the state after 100 breather collisions $(t \sim 88000)$.

After time $\frac{\pi}{\left(V_{1}-V_{2}\right)} \simeq 452.4$, breathers collide. In Fig. 6 one can see breathers at the moment of collision $(t=452.4)$.

The Fourier spectrum of two breathers at $t=452.4$ is shown in Fig. 7.

Finally we show the picture of two breathers after $100 \mathrm{col}-$ lisions at $t \sim 88000$ when they separated again at distance $\simeq \pi$. The initial condition and state after 100 breather collisions are shown in Fig. 8. The Fourier spectrum of that is given in Fig. 9. One can compare it with the initial spectrum in Fig. 5. Low radiation after 100 collisions is shown in Fig. 10, which is a zoomed profile of $|b(x)|$. During numerical simulation the total energy was conserved up to the ninth digit after the decimal point. To ensure the quality of long-term calculations, we performed simulation with different time steps.

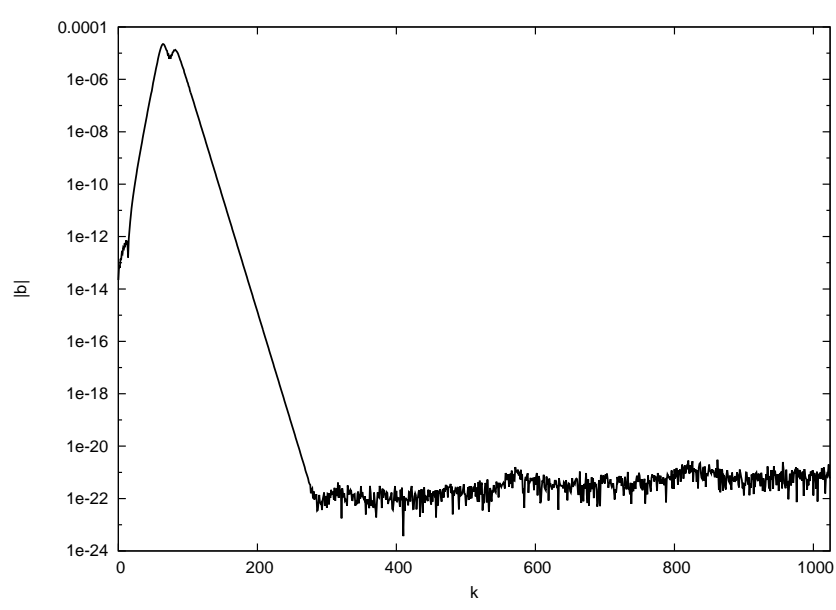

Fig. 9. Fourier spectrum $\left|b_{k}\right|$ after 100 collisions.

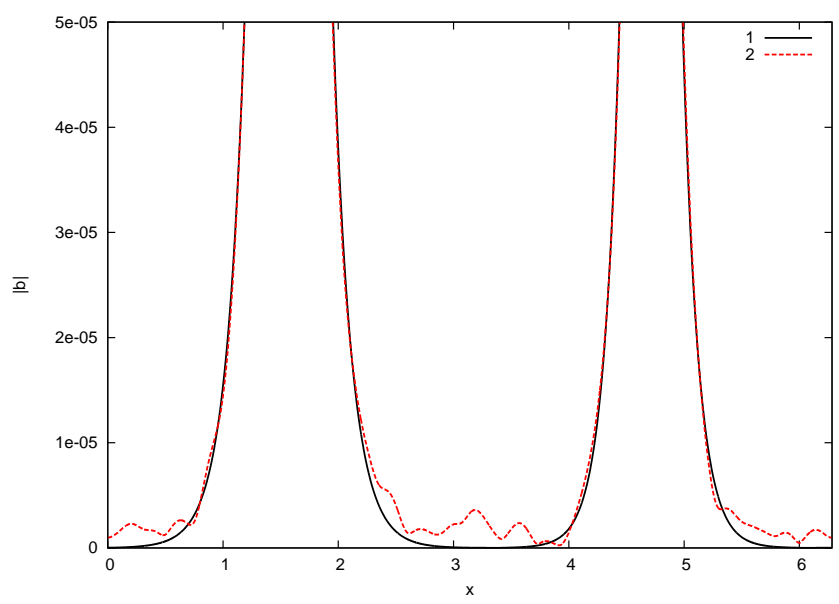

Fig. 10. Zoomed picture from Fig. 8 .

So, the simulation demonstrates that after multiple collisions of breathers, radiation appears. It points to nonelastic collisions and the nonintegrability of Eq. (6).

\section{Conclusions}

We see that individual breathers are not different qualitatively from NLSE solitons. We have studied numerically the interaction of two breathers (solitons) with different values of carrier wave lengths. Interaction of such breathers cannot be described by the NLSE even approximately.

Interaction of such solitons happens to be nonelastic. This experimental fact requires additional study to prove nonintegrability analytically. One can check the 6-wave interaction coefficient on the resonant manifold. It is nonzero if the equation is nonintegrable. 
This new Eq. (6) can be generalized for the "almost" 2D waves or "almost" 3-D fluids. When considering waves slightly inhomogeneous in the transverse direction, one can think in the spirit of the Kadomtsev-Petviashvili equation for the Korteveg-de Vries equation, namely one can treat now frequency $\omega_{k}$ depending on both $k_{x}$ and $k_{y}$ as $\omega_{k_{x}, k_{y}}$, while leaving coefficient $\tilde{T}_{k_{2} k_{3}}^{k k_{1}}$ not depending on $y . b$ now depends on both $x$ and $y$ :

$$
\begin{aligned}
\mathcal{H} & =\int b^{*} \hat{\omega}_{k_{x}, k_{y}} b \mathrm{~d} x \mathrm{~d} y+ \\
& +\frac{1}{2} \int\left|b_{x}^{\prime}\right|^{2}\left[\frac{i}{2}\left(b b_{x}^{* *}-b^{*} b_{x}^{\prime}\right)-\hat{K}_{x}|b|^{2}\right] \mathrm{d} x \mathrm{~d} y .
\end{aligned}
$$

Acknowledgements. This work was supported by Russian Government Grant N11.G34.31.0035 (leading scientist - Zakharov V. E., GOU VPO "Novosibirsk State University"). It was also supported by RFBR Grant 12-01-00943-a and RFBR Grant 12-05-92004HHC_a, the "Fundamental Problems of Nonlinear Dynamics in Mathematics and Physics" program from the RAS Presidium, and "Leading Scientific Schools of Russia" Grant 6170.2012.2.

Edited by: A. Slunyaev

Reviewed by: two anonymous referees

\section{References}

Belokolos, E. D., Bobenko, A. I., Enol'skii, V. Z., Its, A. R., and Matveev, V. B.: Algebrogeometric approach to nonlinear integrable problems, Springer Series in Nonlinear Dynamics, Springer-Verlag, Berlin, 337 pp., 1994.

Dyachenko, A. I. and Zakharov, V. E.: Is free-surface hydrodynamics an integrable system?, Phys. Lett. A, 190, 144-148, 1994.

Dyachenko, A. I. and Zakharov, V. E.: On the Formation of Freak Waves on the Surface of Deep Water, JETP Letters, 88, 307-311, 2008.

Dyachenko, A. I. and Zakharov, V. E.: Compact equation for gravity waves on deep water, JETP Letters, 93, 701-705, 2011.

Dyachenko A. I. and Zakharov, V. E.: A dynamical equation for water waves in one horizontal dimension, European J. Mech. B/Fluids, 32, 17-21, 2012.

Dyachenko, A. I., L'vov, Y. V. and Zakharov, V. E.: Five-wave interaction on the surface of deep fluid, Physica D, 87, 233-261, 1995.
Dyachenko, A. I., Zakharov, V. E., and Kachulin, D. I.: Collision of two breathers at surface of deep water, http://arxiv.org/abs/1201. 4808, 2012.

Fedele, F. and Dutykh, D.: Special solutions to a compact equation for deep-water gravity waves, J. Fluid Mech., 712, 646-660, 2012.

Gardner, C. S., Greene, J. M., Kruskal, M. D., and Miura, R. M.: Method for solving the Korteweg-de Vries equation, Phys. Rev. Lett., 19, 1095-1097,. 1967

Kadomtsev, B. B. and Petviashvili, V. I.: Acoustic turbulence, Soviet Physics Doklady, 18, 115-118, 1973.

Kaup, D. J.: A Higher-Order Water-Wave Equation and the Method for Solving It, Prog. Theor. Phys., 54, 396-408, 1975.

Konopelchenko, B. G.: "Solitons in multidimensions", World Scientific, Singapore, 1993.

Lakoba, T. I. and Yang, J.: A generalized Petviashvili iteration method for scalar and vector Hamiltonian equations with arbitrary form of nonlinearity, J. Comp. Phys., 226, 1668-1692, 2007.

Osborne, A. R.: Nonlinear Ocean Waves and the Inverse Scattering Transform, International Geophysics, 97, 557-569, 2010.

Petviashvili, V. I.: Equation for an extraordinary soliton, Sov. J. Plasma Phys., 2, 257-258, 1976.

Rumpf, B., Newell, A. C., and Zakharov, V. E.: Turbulent Transfer of Energy by Radiating Pulses, Phys. Rev. Lett., 103, 074502, doi:10.1103/PhysRevLett.103.074502, 2009.

Zakharov, V. E.: Stability of periodic waves of finite amplitude on the surface of a deep fluid, J. Appl. Mech. Tech. Phys., 9, 190194, 1968.

Zakharov, V. E.: On Stochastization of one-dimensional chains of nonlinear oscillators, Sov. Phys. JETP, 38, 108-110, 1974.

Zakharov, V. E. and Kuznetsov, E. A.: Optical solitons and quasisolitons, Sov. Phys. JETP, 86, 1035-1046, 1998.

Zakharov, V. E. and Schulman, E. I.: Integrability of nonlinear systems and perturbation theory, in: What is Integrability?, Springer Series Nonlinear Dynamics, Springer-Verlag, Berlin, 185-250, 1991.

Zakharov, V. E. and Shabat, A. B.: Exact theory of two-dimensional self-focusing and one dimensional self-modulation of waves in nonlinear media, Sov. Phys. JETP, 34, 62-69, 1972.

Zakharov, V. E. and Shabat, A. B.: Integration of nonlinear equations of mathematical physics by the method of inverse scattering. II., Funct. Anal. Appl., 13, 166-174, 1979.

Zakharov, V. E., Lvov, V. S., and Falkovich, G.: "Kolmogorov Spectra of Turbulence I", Springer-Verlag, 1992. 